\title{
Archéologie des images et logique rétrospective
}

Note sur le « Manétisme » de Warburg

\section{Andrea Pinotti}

\section{OpenEdition}

\section{Journals}

Édition électronique

URL : http://journals.openedition.org/imagesrevues/3043

DOI : 10.4000/imagesrevues.3043

ISSN : $1778-3801$

Éditeur :

Centre d'Histoire et Théorie des Arts, Groupe d'Anthropologie Historique de l'Occident Médiéval, Laboratoire d'Anthropologie Sociale, UMR 8210 Anthropologie et Histoire des Mondes Antiques

\section{Référence électronique}

Andrea Pinotti, « Archéologie des images et logique rétrospective », Images Re-vues [En ligne], Horssérie 4 | 2013, document 16, mis en ligne le 30 janvier 2013, consulté le 30 janvier 2021. URL : http:// journals.openedition.org/imagesrevues/3043; DOI : https://doi.org/10.4000/imagesrevues.3043

Ce document a été généré automatiquement le 30 janvier 2021.

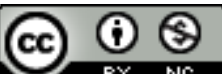

Images Re-vues est mise à disposition selon les termes de la Licence Creative Commons Attribution Pas d'Utilisation Commerciale 4.0 International. 


\section{Archéologie des images et logique rétrospective}

Note sur le « Manétisme » de Warburg

\section{Andrea Pinotti}

1 Le Vingtième siècle a connu un «cézannisme», en littérature (Rilke, D.H. Lawrence, Gertrud Stein, Hemingway, Virginia Woolf, pour ne citer que quelques noms) comme en philosophie (MerleauPonty, Heidegger, Lyotard, Deleuze, Maldiney). On a utilisé Cézanne pour «penser en peinture», pour penser en image ce qui ne se laissait penser autrement, ce qui ne pouvait pas être

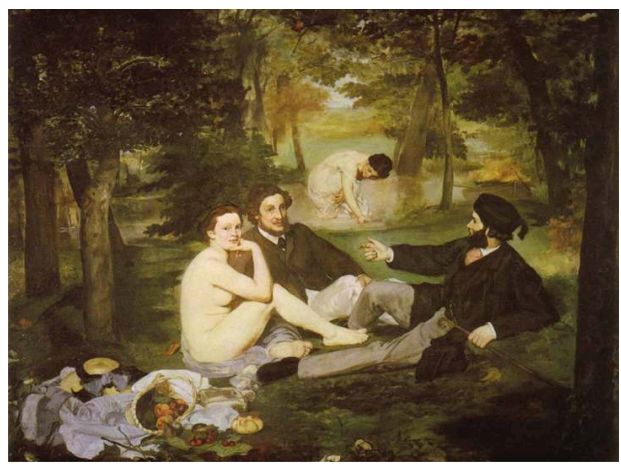
pensé avec les concepts². Mais ce siècle a aussi connu un «manétisme», dont Aby Warburg constitue un représentant éminent. On tentera ici d'en rendre compte à partir de la caractérisation traditionnelle de Manet comme peintre de la rupture.

2 Baudelaire qui, malgré l'admiration qu'il éprouvait pour son ami Manet (une «profonde correspondance», selon Valéry) ${ }^{3}$ ne l'avait pas identifié comme le "peintre de la vie moderne», lui avait lancé, dans une lettre de 1865, une formule destinée à devenir fameuse : «Vous n'êtes que le premier dans la décrépitude de votre art» ${ }^{4}$. Mais, ce qui apparaissait à Baudelaire comme une «décrépitude» inaugurée par Manet allait justement prendre, dans les années suivantes, la signification d'un nouvel horizon pictural, un horizon ouvert par un scandale.

3 On connaît les cris horrifiés qui avaient accueilli le Déjeuner sur l'herbe [FIG. 1], exposé en 1863 dans un Salon-Annexe, parallèle au Salon officiel. 
Fig.1

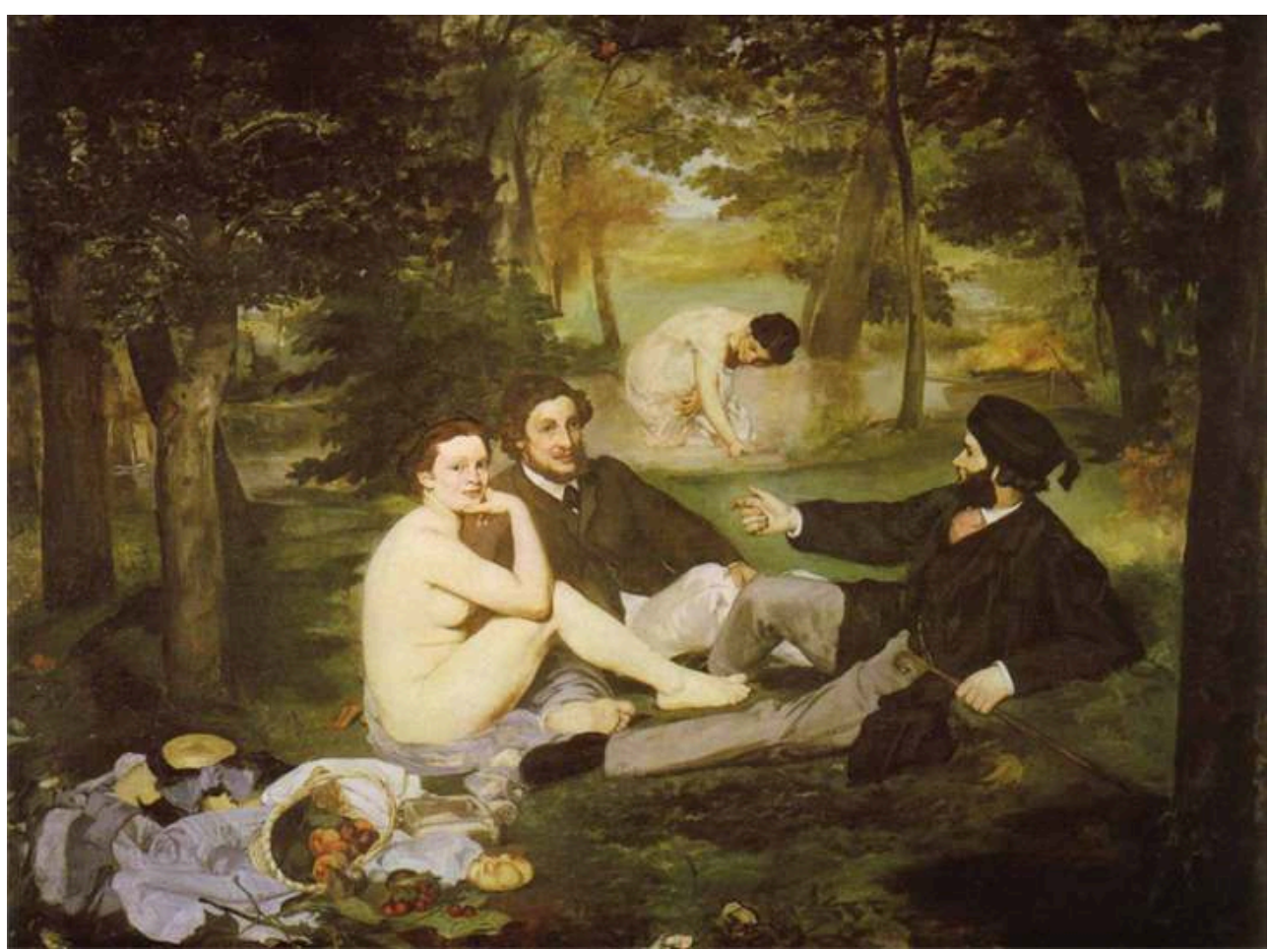

Edouard Manet, Le déjeuner sur I'herbe (titre originaire: Le Bain), 1863, huile sur toile, Paris, Musée d'Orsay.

4 Ils avaient été précédés par une "véritable émeute» quand la toile avait été dévoilée, avant l'ouverture du Salon, à la galerie Martinet. Quatre ans plus tard, Émile Zola se souvient de la réaction du public et la commente en ces termes : «Des sifflets et des huées, comme il est d'usage, annoncèrent qu'un nouvel artiste original venait de se révéler $»^{5}$. Néanmoins, tout en remarquant qu'une femme nue en compagnie d'hommes habillés n'était pas chose tout à fait inhabituelle (au Louvre on pouvait repérer "plus de cinquante tableaux» similaires), il explique que l'intention de Manet n'était certes pas de susciter un esclandre facile au moyen d'un sujet si grossièrement obscène : «Les peintres, surtout Édouard Manet, qui est un peintre analyste, n'ont pas cette préoccupation du sujet qui tourmente la foule avant tout ; le sujet pour eux est un prétexte à peindre, tandis que pour la foule le sujet seul existe. Ainsi, assurément, la femme nue du Déjeuner sur l'herbe n'est là que pour fournir à l'artiste l'occasion de peindre un peu de chair» ${ }^{6}$.

5 Neuf, original aux yeux de Zola, Manet l'est non pour ce qu'il peint, mais plutôt pour la manière dont il peint : «L'artiste ne peint ni l'histoire ni l'âme ; ce qu'on appelle composition n'existe pas pour lui» ${ }^{7}$. Il ne doit pas être jugé comme un moraliste ou un écrivain qui transpose en image un contenu élaboré ailleurs : il est un peintre, «il sait peindre, et voilà tout» ${ }^{8}$. Par ces mots, Zola inaugure, en 1867, une lignée interprétative qui fait de Manet la figure d'une césure entre le passé (la peinture du quoi, du sujet, illustrative et narrative) et le futur (la peinture du comment, consacrée exclusivement à la mise en œuvre de ses propres moyens, à la composition de lignes et de couleurs), lignée reprise et approfondie durant le $\mathrm{XX}^{\mathrm{e}}$ siècle par les théoriciens de la peinture, en particulier par ceux qui considèrent l'art dit abstrait ou encore non-figuratif comme ne 
se rapportant à rien d'autre qu'à lui-même, puisqu'il a supprimé tout référent extérieur.

Dans le sillage de Zola, quelques années plus tard, Stéphane Mallarmé confirme l'idée qu'il y a une nouveauté absolue dans la peinture de Manet : dans un article publié originairement en anglais, "The Impressionists and Edouard Manet», il parle de la «supreme originality» d'un «bold innovator», de ses «new laws of space and light». Et il conclut : Manet «seems to ignore all that has been done in art by others» ${ }^{9}$.

7 En raison de ce rôle révolutionnaire, Manet ne manque pas d'attirer l'attention des philosophes intéressés par le domaine de la figuration : Michel Foucault, par exemple, dans une conférence dédiée au peintre en 1971, parle d'une «rupture en profondeur» à propos de l'art de Manet, expression prégnante, si l'on pense à l'insistance qui est la sienne sur la question des continuités et des ruptures des paradigmes représentationnels : «Manet est celui qui pour la première fois dans l'art occidental, au moins depuis la Renaissance, au moins depuis le Quattrocento, s'est permis d'utiliser et de faire jouer, à l'intérieur même de ses tableaux, à l'intérieur même de ce qu'ils représentaient, les propriétés matérielles de l'espace sur lequel il peignait» ${ }^{10}$.

Reconduire le tableau à ses propriétés matérielles signifie dénoncer son caractère de chose, tout en suspendant le tacite pacte illusionniste signé avec l'observateur: Manet «réinvente, ou peut-être invente-t-il, le tableau-objet, le tableau comme matérialité, comme chose colorée que vient éclairer une lumière extérieure et devant lequel ou autour duquel vient tourner le spectateur». Et c'est justement la question d'une source de lumière externe, qui se répand sur le tableau comme sur n'importe quel autre objet non artistique accroché au mur - un veston suspendu à un portemanteau, une casserole pendue à un clou - qui tend à assumer une signification centrale dans le Déjeuner sur l'herbe: commentant cette œuvre, Foucault souligne le conflit, au sein du tableau, entre une source de lumière traditionnelle, une source lumineuse interne au tableau provenant d'en haut à gauche, et une source externe, frontale et perpendiculaire : «Deux systèmes discordants et hésitants d'éclairage en profondeur».

Or, en 1955 déjà, Georges Bataille avait insisté sur la fonction de rupture de la peinture (bien qu'encore figurative) de Manet : «Le nom de Manet - ainsi commence son essai - a dans l'histoire de la peinture un sens à part. Manet n'est pas seulement un très grand peintre : il a tranché avec ceux qui l'ont précédé; il ouvrit la période où nous vivons»" ${ }^{11}$. Il soulignait ainsi l'indifférence souveraine pour le sujet, la négation de l'éloquence et des significations, le dépassement des aspirations illusionnistes tridimensionnelles de la surface picturale, le rejet, enfin, de tout ce qui, en quelque façon, était extra-pictural.

10 Autrement dit, une suppression du contenu dont André Malraux, plus tôt encore, en 1951, avait fait état dans Les voix du silence: «L'Exécution de Maximilien de Manet, c'est le Trois Mai de Goya, moins ce que ce tableau signifie [...]. L'orientation que Manet tentait de donner à la peinture rejetait ces significations» $»^{12}$. Selon Malraux, Manet marquerait «l'origine de la peinture moderne»: «Pour que la tradition picturale soit déchirée comme l'avait été la tradition littéraire par les grands poètes au début du siècle, il faut attendre Manet $»^{13}$.

11 On retrouve une interprétation similaire de la nouveauté de Manet chez les théoriciens anglo-saxons. D'abord chez Clement Greenberg qui, dans l'essai intitulé Modernist Painting (1960) parle de la même manière d'une césure, d'un nouveau commencement 
de l'art: «Manet's became the first Modernist pictures by virtue of the frankness with which they declared the flat surfaces on which they were painted $»^{14}$.

Et même Michael Fried qui - malgré son héritage greenberguien - a consacré en 1969 un long article aux «sources» de Manet, donc à ce que Manet reprend à ceux qui l'ont précédé en termes de «sujet», admet finalement qu'il faut parler, à propos de cet artiste, d'un nouveau genre de peinture s'identifiant avec la peinture tout court : «the unity that I have called painting altogether». Dans cette interprétation, c'est encore une fois le même tableau qui joue un rôle crucial : «Manet's most ambitious paintings of the first half of the 1860s [...] may perhaps be thought of as constituting a new genre of painting, which in effect sought to comprehend, and thereby to supersede, all the others. The Déjeuner sur l'herbe represents a kind of culmination of this development, being at once landscape, portrait, and still life - to say nothing of the implications of its use of previous art ${ }^{15}$.

Contre la lecture formaliste et moderniste de Manet (plus précisément contre celle de Bataille, interprète de Manet), Rosalind Krauss s'est durement exprimée en 1986. Il est vrai que Bataille, tout en s'éloignant apparemment de sa propre théorie de l'image, avait souligné le rôle du peintre en tant qu'initiateur de l'autonomie de l'art pur, ce qui mécontentait profondément Krauss : «One turns the pages of Georges Bataille's book on Manet with a mounting sense of disappointment. Is it really Bataille who is telling usonce again-that Manet's achievement was the destruction of subject matter so that in its place, from among its ruins, should arise pure painting-"painting," as he writes, "for its own sake, a song for the eyes of interwoven forms and colors"? $»^{16}$.

Mais la désillusion laisse bientôt place à une revendication anti-moderniste, et de Bataille et de Manet, rattaché quant à lui par Krauss à un commencement tout à fait différent: Manet «is the beginning of modern art, an art that celebrates the silent autonomy of vision. But behind this beginning is another, wholly alternate beginning ${ }^{17}$. Il s'agit là d'une alternative au modernisme qui n'appartient pas à la «modernist fetishization of sight» ${ }^{18}$, mais qui est plutôt «"blinding," "sight-destroying," and in which representation dares to be neither appropriative nor productive» ${ }^{19}$.

Dix ans plus tard, dans Formless (1997), Rosalind Krauss et Yves-Alain Bois prennent une fois encore Manet comme point de départ d'une lecture désormais célèbre, vouée à déconstruire l'opposition même de la forme et du contenu et, par là même, à sauver Bataille de l'interprétation formaliste en revendiquant son originalité dans le domaine de l'«informe». Et Bois écrit : «Bataille wrote of Manet: “To break up the subject and reestablish it on a different basis is not to neglect the subject; so it is in a sacrifice, which takes liberties with the victim and even kills it, but cannot be said to neglect it." It is this type of alteration that we want both to describe and to attempt ${ }^{20}$. La perspective est différente donc, mais Manet est toujours reconnu comme un point de départ

Et pourtant Bataille, à bien y regarder, n'avait pas seulement parlé de rupture à propos de l'art de Manet. Il s'était en outre arrêté sur une caractéristique «étrange» du peintre parisien : «Un des aspects les plus étranges de l'art de Manet tient à ses emprunts. Plusieurs fois, Manet emprunta la donnée schématique ou d'un tableau, ou d'une gravure ancienne. Nous savons depuis longtemps que la composition du Déjeuner sur l'herbe est donnée dans Le Jugement de Pâris de Raphaël, que Marc-Antoine a gravé» ${ }^{21}$. Pourquoi cet italique, «emprunta»? Comme c'est le cas avec la Venus d'Urbino de Titien, prise pour modèle de l'olympia, il s'agit aussi, dans le Déjeuner, d'une «métamorphose», d'un "passage d'un monde à l'autre»"22: "Le Déjeuner sur l'herbe était lui-même la 
négation de ce Concert champêtre [Fig. 2] où Giorgione, s'il avait introduit des femmes nues près des musiciens de la Renaissance, l'avait fait sous le bénéfice de la fable grecque. Manet ne tira du tableau du Louvre que le thème (il tira, comme je l'ai dit, le schéma graphique de son tableau d'une gravure...).

Fig.2

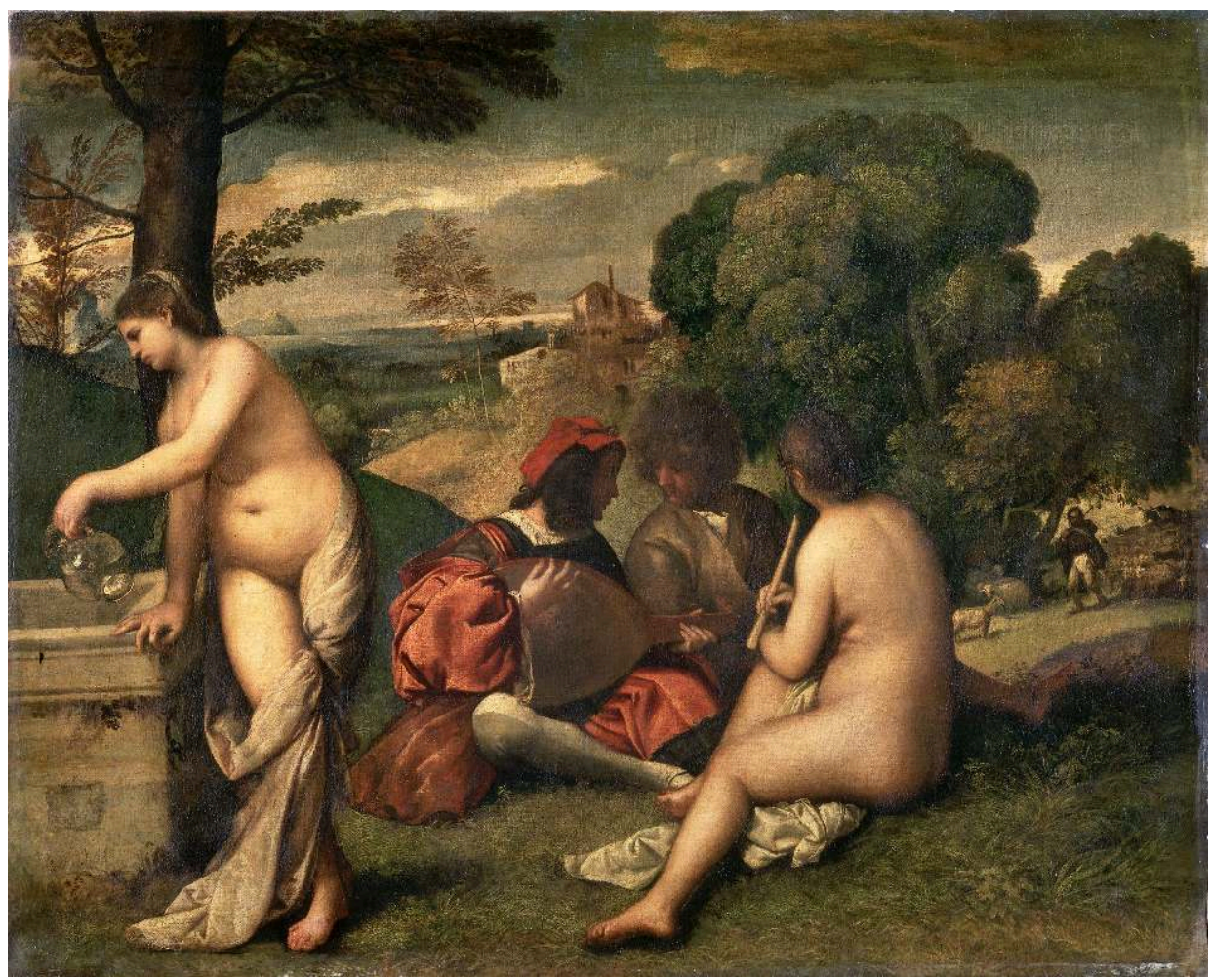

Giorgione/Titien, Le concert champêtre, vers 1509, huile sur toile, Paris, Musée du Louvre

Mais dans le thème et le schéma, l'un et l'autre mythologiques, il introduisit le monde présent, trouvant dans ce changement ce qu'il voulait, le renversement du passé, la naissance d'un ordre nouveau» ${ }^{23}$.

Emprunter signifie dès lors reprendre, non pour imiter, répéter, confirmer, mais plutôt pour transformer, varier, et, à la limite inverser. Continuer pour rompre ou rompre pour continuer?

\section{Continuité}

« Nous savons depuis longtemps... » disait Bataille en 1955. Depuis quand, au juste, saiton quels furent les modèles de Manet pour son Déjeuner? Depuis toujours. Exposé en 1863, le tableau était, dès l'année suivante, reconduit par Ernest Chesneau à ses origines classiques, à un Jugement de Pâris (perdu) de Raphaël, gravé par Marcantonio Raimondi [Fig.3] : «Il est à peine croyable que M. Manet ait emprunté à Raphaël une de ses compositions. Cela n'est, hélas! que trop vrai cependant. Que l'on compare son Déjeuner sur l'herbe à certain groupe du Jugement de Pâris»» 
FIg.3

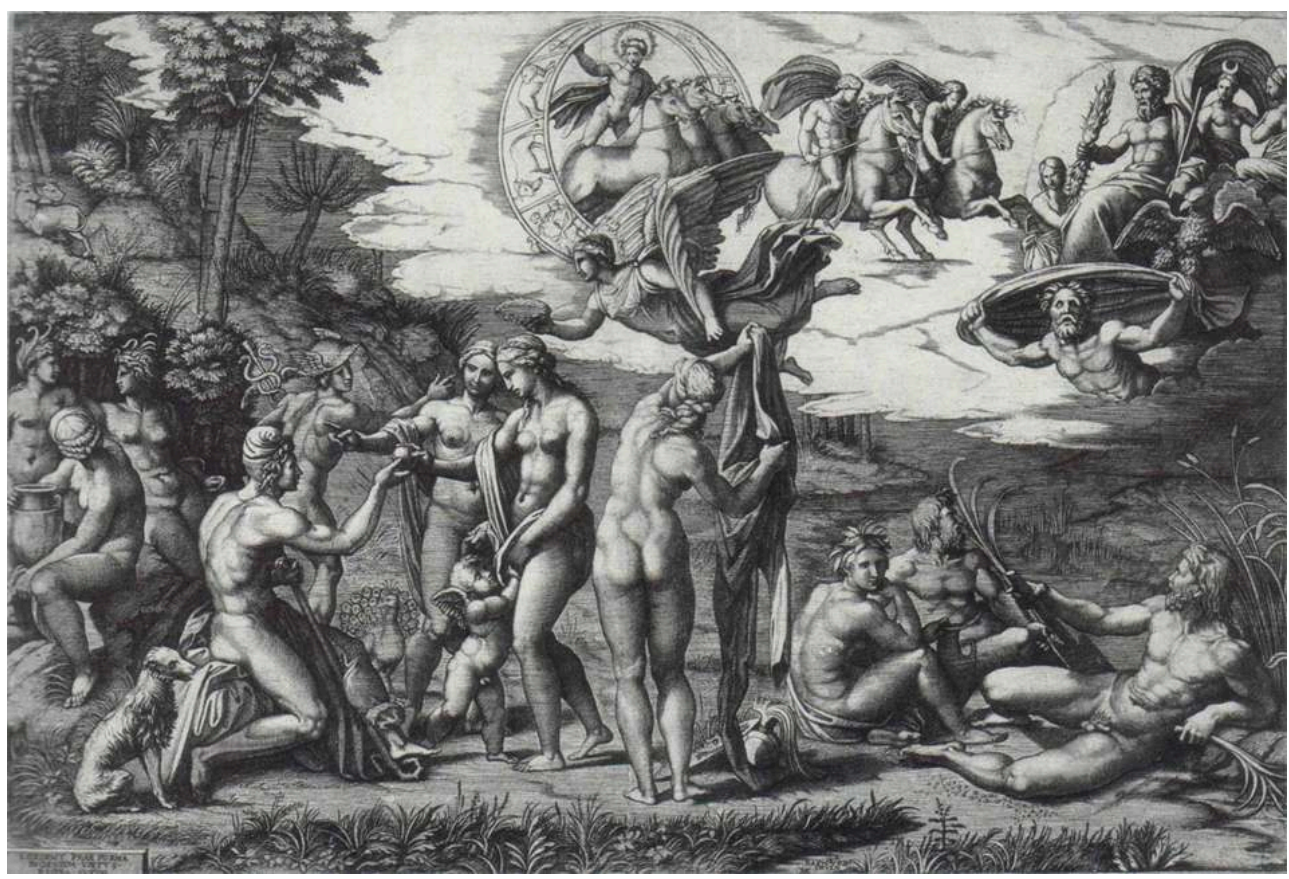

Marcantonio Raimondi, Le jugement de Pâris, 1515-1516, gravure après un dessin perdu de Raphaël

Cette ascendance fut confirmée et approfondie en 1908 par Gustav Pauli, le futur directeur de la Kunsthalle de Hambourg (nommé successeur d'Alfred Lichtwark, de 1914 à 1933), dans un article intitulé Raffael und Manet ${ }^{25}$.

21 Et c'est à Pauli qu'Aby Warburg se rattache d'emblée, en 1929, quand il dicte à Gertrud Bing des notes très denses sur le Déjeuner. Il est alors bien conscient de se livrer à une opération délicate : «De tous les tableaux modernes -admet-il en ouverture-, aucun ne présente davantage de difficultés au critique d'art soucieux d'établir le rôle déterminant, essentiel, des rapports formels et thématiques avec la tradition, que Le Déjeuner sur l'herbe de Manet» ${ }^{26}$. Pourtant, il ne renonce pas à l'entreprise, s'appuyant d'abord sur le fait que Manet lui-même ${ }^{27}$ s'était référé au Concert champêtre (une œuvre qui, en son temps, était attribuée à Giorgione, alors qu'aujourd'hui la majorité des critiques tend à l'assigner à Titien) comme au modèle - le «Vorbild», comme le dit Warburg - justifiant sa propre composition. Dès les premiers paragraphes, Warburg dévoile ses cartes, en expliquant ce que les notions d'originalité et de nouveauté signifient pour lui, en ce qui concerne l'art en général, et, plus particulièrement en ce qui concerne Manet : «Manet, l'homme qui s'avançait vers la lumière, avait-il besoin telle est la question que nous posons aujourd'hui - de se poser par ce retour en arrière en administrateur fidèle de l'héritage du passé, lui dont la figuration immédiate apprenait au monde qu'on ne saurait prétendre, sans prendre part au patrimoine universel de l'esprit, trouver un style créateur de nouvelles valeurs expressives, dès lors que celles-ci puisent leur force de pénétration non pas dans le rejet des formes anciennes, mais dans les écarts subtils induits par leur transformation ?» ${ }^{28}$.

22 Jusqu'alors, Warburg parlait du rapport essentiel et organique de Manet (comme de chaque artiste digne de ce nom) avec l'histoire de l'art et la tradition, mais non de la manière dont un tel rapport était rendu effectif. «Vorbild», tel est le terme qui qualifie le Concert champêtre de Giorgione comme modèle du tableau de Manet. C'est-à-dire en 
tant qu'image (Bild), tout à la fois placée devant les yeux de l'artiste qui s'en inspire pour son œuvre (si on entend vor dans un sens spatial) et ayant existé dans une époque antérieure à celle de l'image qui en dérive (si l'on entend vor dans un sens temporel).

Mais ce Vorbild de Giorgione n'est pas le premier modèle, et Raphaël et Marcantonio (dont la gravure a inspiré, à son tour, une décoration pariétale réalisée autour de 1630, aujourd'hui conservée à la Villa d'Este à Tivoli [Fig. 4]) ne sont eux-mêmes que des médiateurs (Vermittler)29: il faut remonter plus haut, le long de la chaîne des images, pour identifier l'origine de la série.

FIg.4

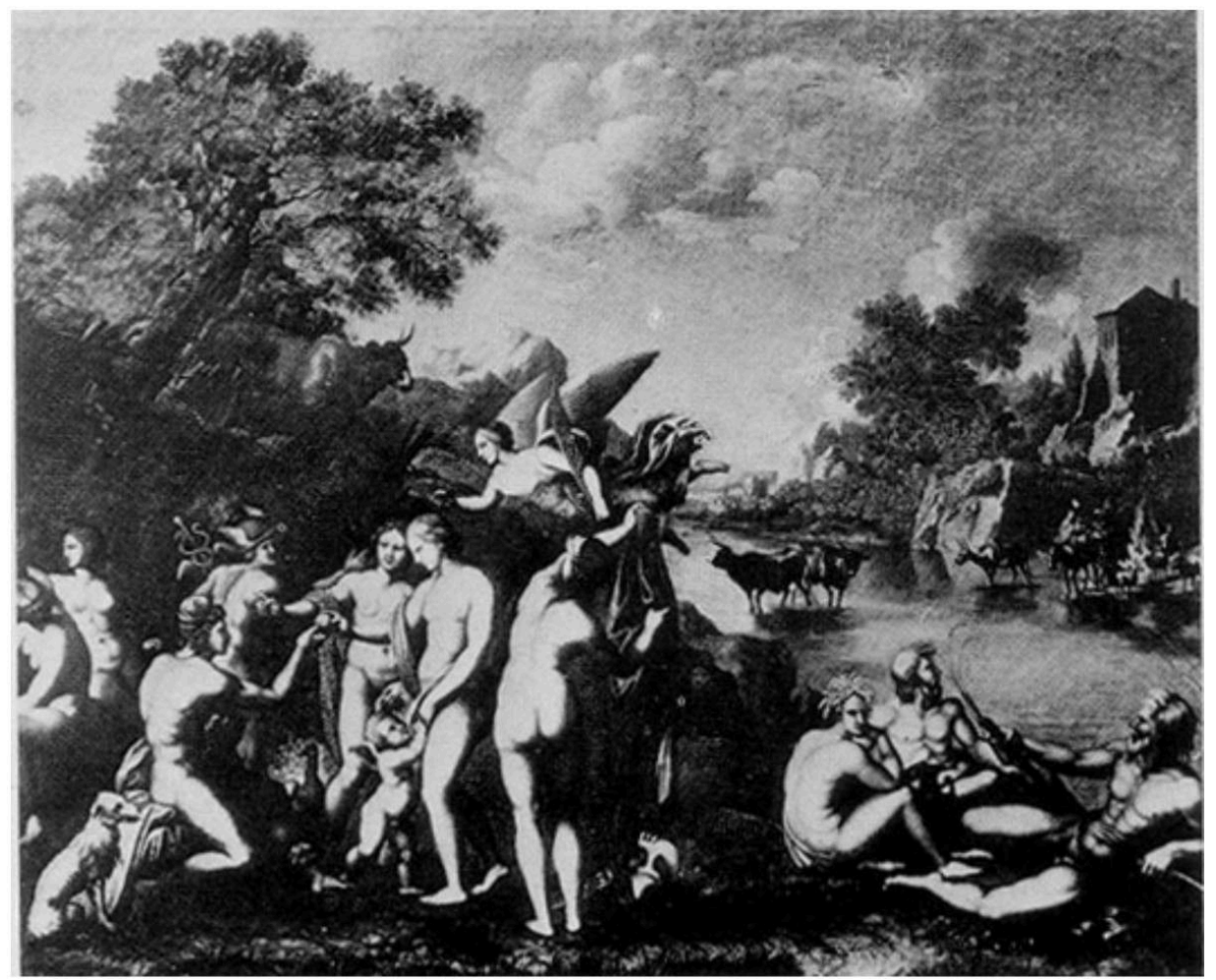

Nicolaes Berchem, Le jugement de Pâris, vers 1650, décoration pariétale, après la gravure de Marcantonio Raimondi, Tivoli, Villa d'Este 
Fig.5

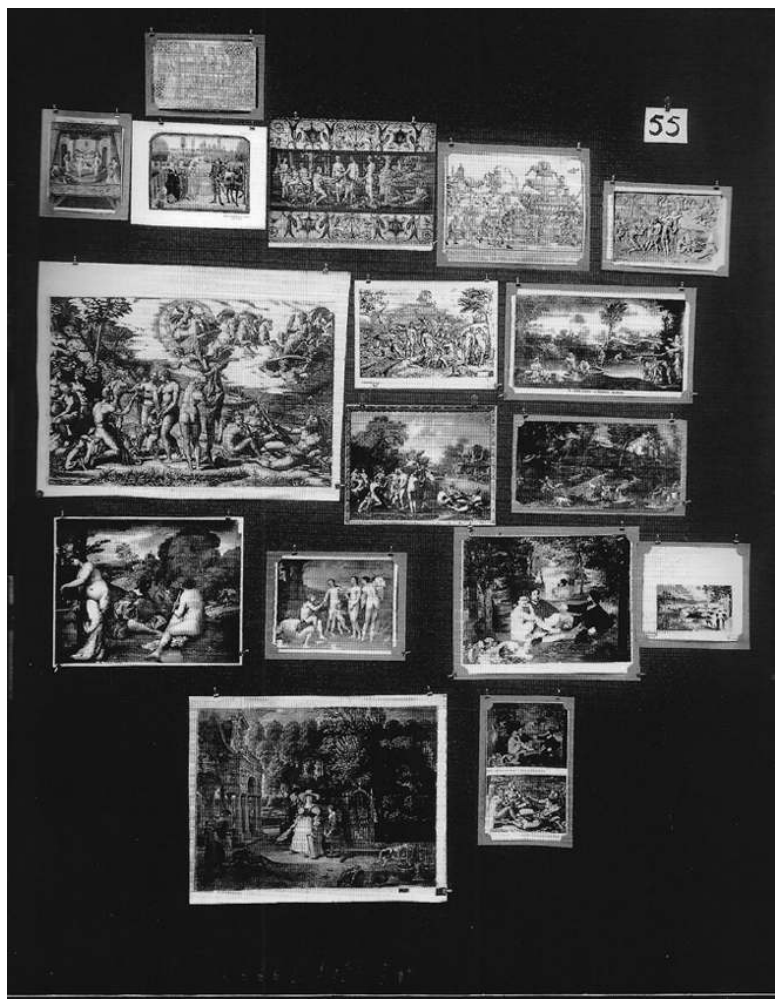

Aby Warburg, Der Bilderatlas Mnemosyne, éd. par M. Warnke, Berlin, Akademie Verlag, 2000, planche 55

Et c'est précisément avec le Déjeuner, cette œuvre devenue célèbre comme point de rupture de la tradition et comme césure du continuum des images que l'on peut retourner à la strate primitive, et indiquer le modèle antique («das antike Vorbild») "avec une exactitude qui reste exceptionnelle dans la science de l'art» ${ }^{30}$. Il s'agit de deux bas-reliefs qui appartiennent à des sarcophages hellénistiques représentant le Jugement de Pâris ${ }^{31}$ : l'un est muré dans la façade de l'Académie de France, à la Villa Médicis [Fig.6], l'autre est inséré, également à Rome, dans la façade du Casino de la Villa Doria Pamphili [Fig.7 $]^{32}$.

FIg.6

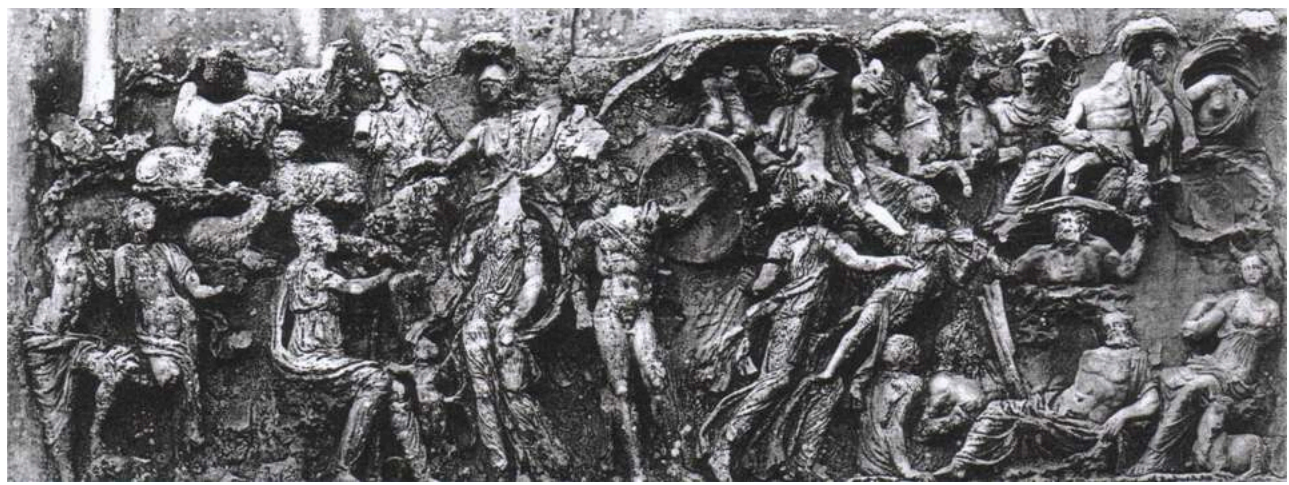

Le jugement de Pâris, sarcophage romain, II siècle, Rome, Villa Médicis. 
Fig.7

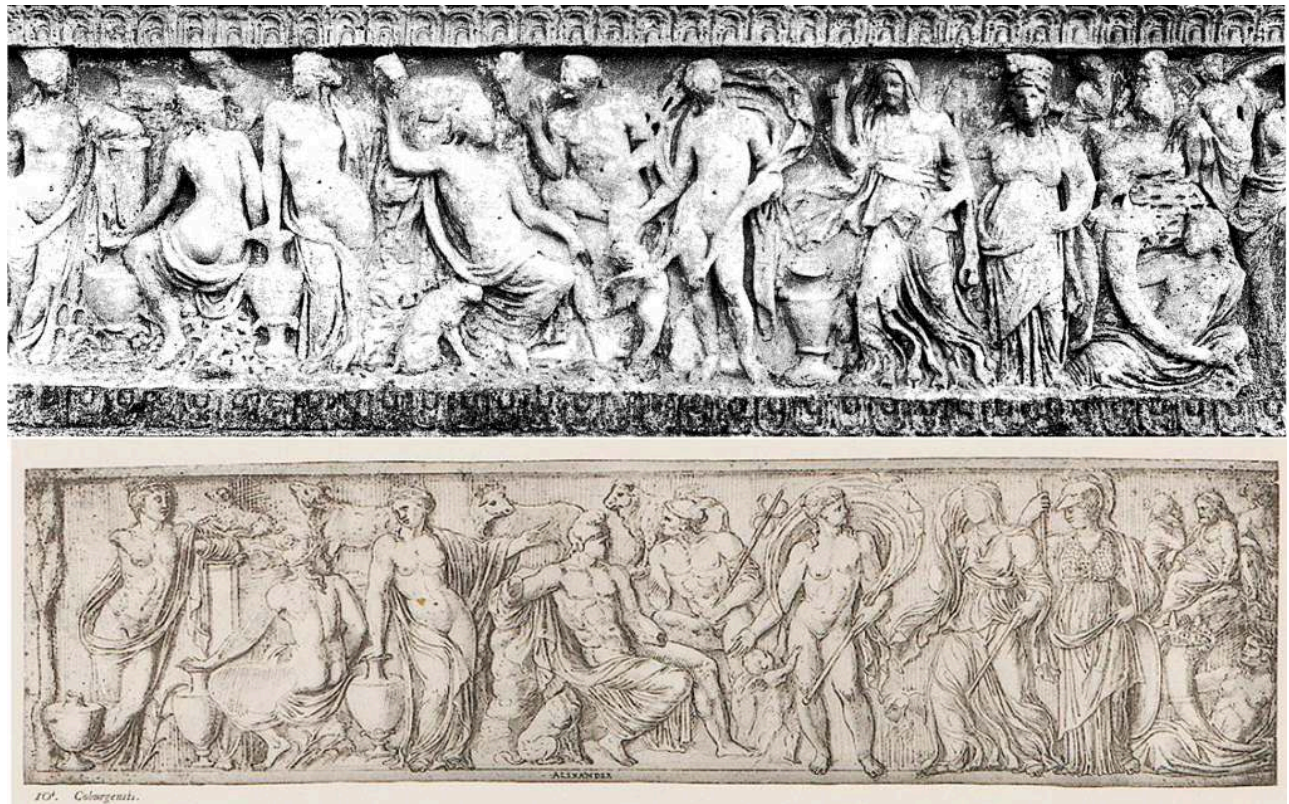

En haut : Le jugement de Pâris, sarcophage romain, II siècle, Rome, Villa Doria Pamphili ; en bas : reconstruction graphique du Codex Coburgensis, dans C. Robert, Die antiken Sarkophagreliefs, Berlin, Grote - Gebr. Mann, 1890, vol. II (Mythologische Cyklen), planche IV, ill. 10'.

Fig.8

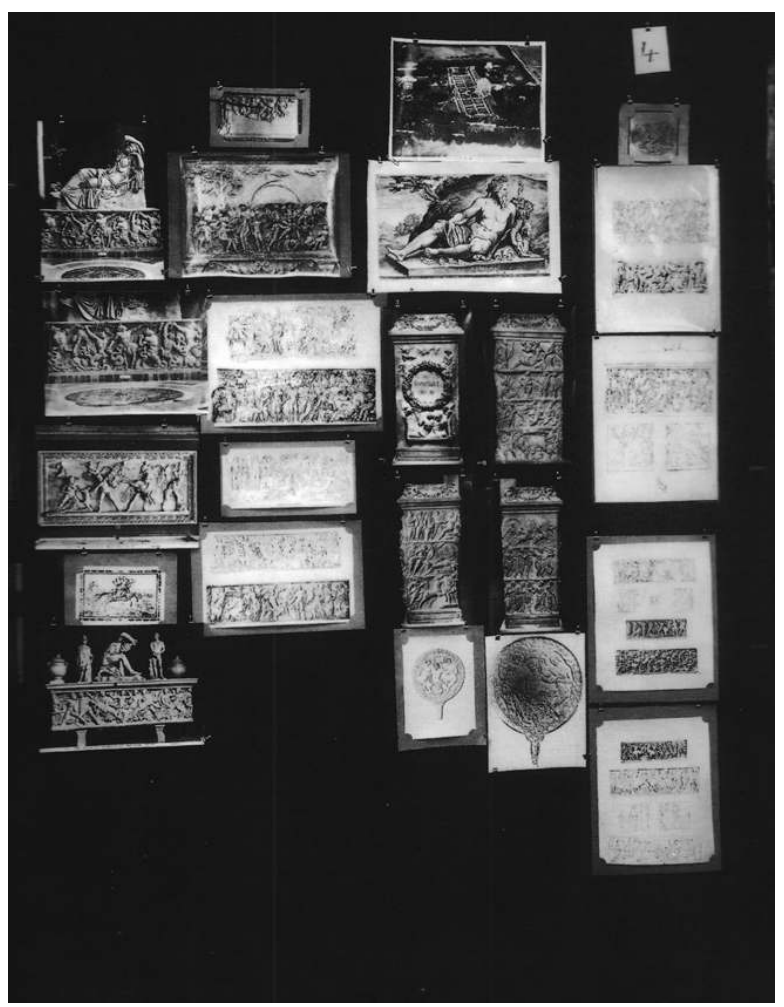

Aby Warburg, Der Bilderatlas Mnemosyne, éd. par M. Warnke, Berlin, Akademie Verlag, 2000, planche 4

Dans son article, Pauli mentionnait les historiens de l'art qui avaient indiqué une telle dérivation : Otto Jahn, en $1849^{33}$ et Anton Springer, en $1878^{34}$. Mais Henry Thode ${ }^{35}$ a 
rappelé que Sandrart, dès 1675 , avait explicité la relation reliant Marcantonio et Raphaël avec le sarcophage de Villa Médicis : « ... das Urtheil des Paris, [...] nach Inhalt des bekandten Antichen Basso Rilievo, so noch bey dem Palazzo di Medici in Rom zu sehen ist $»^{36}$.

Cependant, à l'occasion d'une analyse plus approfondie, Warburg observe qu'il n'y a pas simple répétition d'un modèle iconographique à travers les siècles. Si l'on adopte un regard «micrologique», on peut observer «des écarts en apparence insignifiants [anscheinend ganz unbedeutenden Abweichungen] dans le jeu des gestes et du visage», capables d'opérer une véritable "inversion énergétique de l'humanité représentée»" Sur le bas-relief, les regards des demi-dieux sont orientés avec crainte vers le ciel («Phobos cultuel», dit Warburg, propre à un motif funéraire), tandis que dans le dessin de Raphaël gravé par Raimondi, le regard de la figure à gauche du groupe est explicitement tourné vers le spectateur en signe de foi terrestre, selon une orientation reprise plus tard par Manet lui-même. Il s'agit d'un choix intentionnel, comme le prouve une autre gravure sur le même thème de Giulio Bonasone [Fig. 9] (un artiste contemporain de Raimondi), évoquée par Warburg : malgré l'inversion latérale droitegauche, l'artiste reste fidèle au modèle antique, tout en sauvegardant l'orientation verticale des regards vers le ciel.

FIg.9

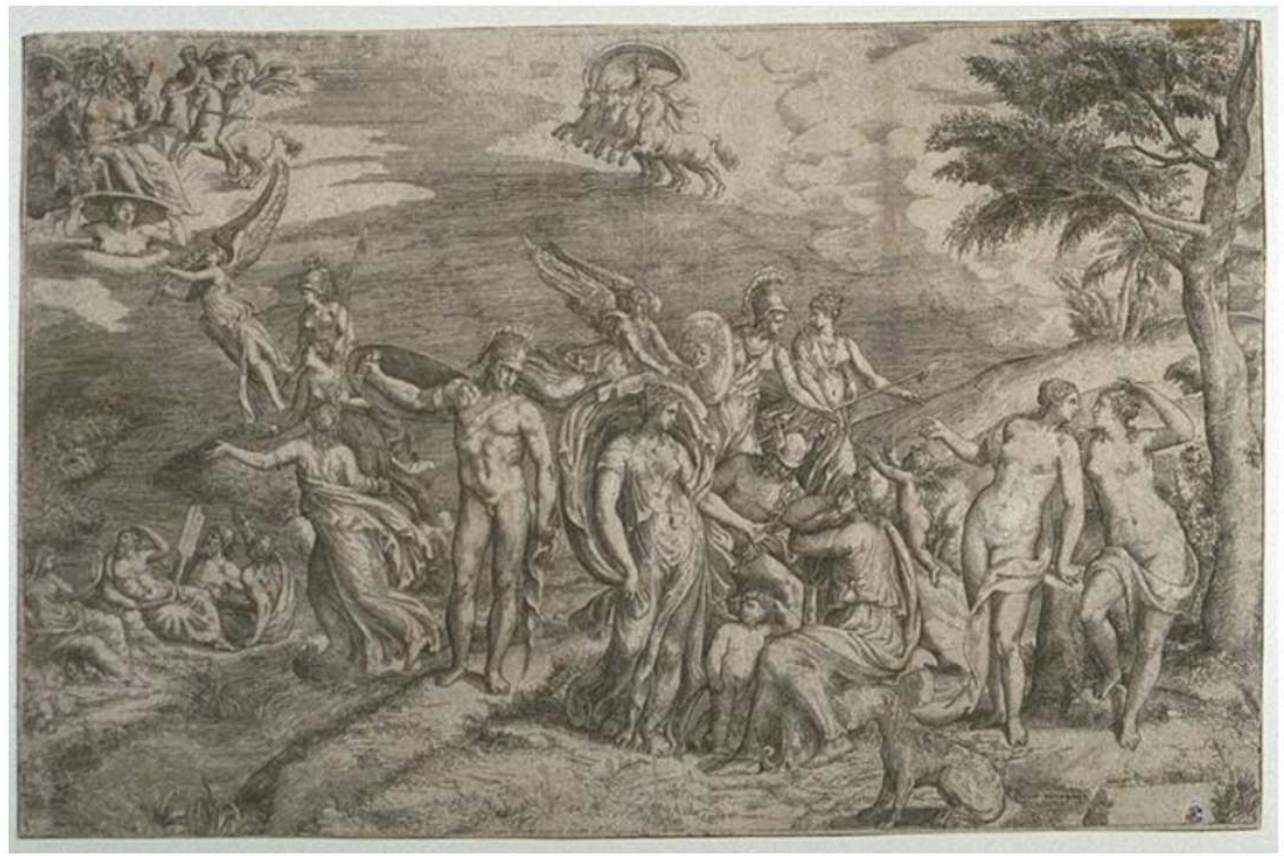

Giulio Bonasone, Le jugement de Pâris, gravure, 1565 ca.

Warburg lui-même est donc obligé d'admettre que - malgré l'éclatante «exactitude» de la reconstruction de la généalogie iconographique établie quelques lignes au-dessus, malgré la «composition typiquement antiquisante» de la gravure de Raimondi - celle-ci n'est pas du tout la «reproduction fidèle $\|^{38}$ du sarcophage ancien. Bien au contraire : elle «s'écarte sur ce point du schéma antique» ${ }^{39}$. Le regard du personnage dirigé vers le spectateur est explicitement défini comme un "caractère non antique» ${ }^{40}$. Ceci est indirectement démontré par un autre exemple, un sarcophage de la Villa Ludovisi : ceux qui ont cru à l'authenticité de la nymphe (déjà disparue du relief au temps de 
Warburg, mais reproduite dans des dessins et gravures [Fig.10]) dont le regard se tournait vers l'extérieur, se sont trompés : il s'agissait d'une «falsification» ${ }^{41}$.

FIg.10

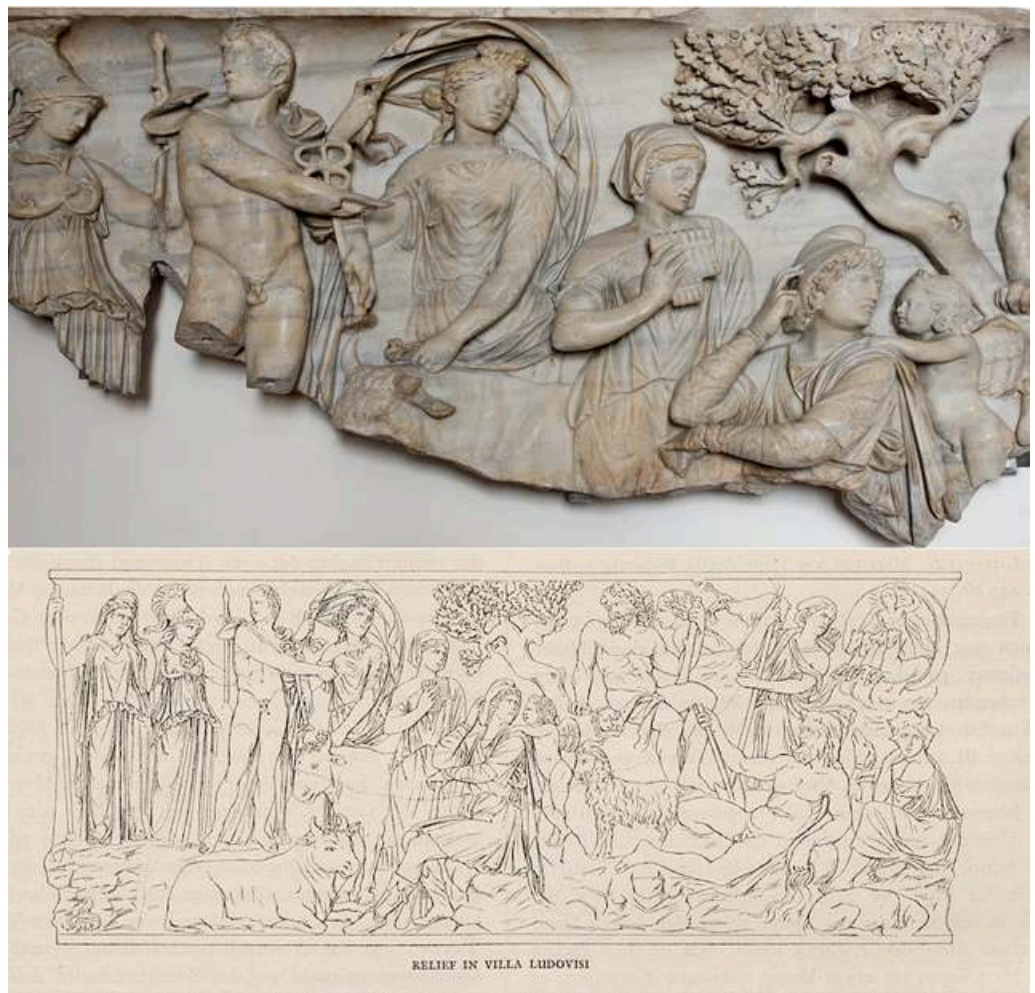

En haut : Le jugement de Pâris, fragment d'un sarcophage romain, II siècle, Rome, Museo Nazionale di Palazzo Altemps (collection Ludovisi) ; en bas : reconstruction graphique dans C. Robert, Die antiken Sarkophagreliefs, Berlin, Grote - Gebr. Mann, 1890, vol. II (Mythologische Cyklen), p. 17

Si l'on prend en compte ce détail crucial de l'orientation du regard dans la constellation d'images constituant l'ensemble des antécédents du Déjeuner, il appert que l'Antiquité ne se situe pas «vor» (devant et avant) sa redécouverte moderne: l'image de la Renaissance, tout en s'«inspirant» du prototype hellénistique, ne le répète pourtant pas. Ici (mais le discours pourrait être confirmé pour d'autres reconstitutions de chaînes d'images opérées par Warburg, également lacunaires), le modèle en tant que Vorbild se caractérise plus par son absence que par sa présence. Il en résulte que le prototype antique n'est pas moins construit que reconstitué. Selon un mouvement de rétroaction, le Vorbild se révèle en même temps Nachbild: le proto-type comme prétype, comme pré-image, se présente aussi comme une post-image, instituée a posteriori par l'artiste moderne. Ainsi, l'Antiquité s'avère autant un modèle pour la Renaissance que le fruit de son invention, ce qui manifeste la puissance productive et pas seulement reproductive de la mémoire, ce qui montre la véritable poiesis de Mnemosyne. L'inventaire des pré-formations («Inventar der Vorprägungen») de la conscience collective de l'imaginaire occidental, que Warburg entendait fournir dans son projet titanesque et inachevé de l'atlas Mnemosyne, doit donc être également compris comme un répertoire de post-formations. 


\section{L'après-coup}

manière convaincante sur les implications différentielles du Nachleben comme répétition iconique, sur le fait que ce qui se répète n'est pas le même, mais ce qui diffère. À cet égard, ses références privilégiées sont le Deleuze de Différence et répétition («il n'y a que l'étrange qui soit familier, et seulement la différence qui se répète» ${ }^{42}$ ) et surtout Freud. Il y a, selon Didi-Huberman, une analogie étroite entre la manière de penser le temps de l'origine, propre à la conception warburghienne de la «survivance des images», et la conception freudienne de la postériorité : «La Nachträglichkeit freudienne serait exactement à la mémoire des "traumatismes" affectant l'histoire des symptômes ce que le Nachleben warburgien est à la mémoire des "sources" affectant l'histoire des images. Dans les deux cas, en effet, l'origine ne se constitue que dans le retard de sa manifestation $»^{43}$.

C'est dans l'Esquisse d'une psychologie scientifique que, pour la première fois, on rencontre en quelque sorte l'idée d'un Nachbild qui se projetterait sur le Vorbild, le constituant par là comme tel. Didi-Huberman résume en ces termes cette découverte : "Cela se passe vers 1895 : cette année-là, Freud comprend que l'origine n'est pas à penser comme un point fixe, fût-il haut placé dans la ligne du devenir. L'origine ne cesse de se feuilleter : vers le passé, bien sûr, mais aussi vers le futur, si l'on peut dire. La grande hypothèse de Freud sur le temps psychique prend ici toute sa mesure. Elle s'incarne dans la notion, capitale et paradoxale, de l' "après-coup" (Nachträglichkeit). Elle suppose, en toute formation de l'inconscient - particulièrement dans le symptôme hystérique - un processus intervallaire que Freud aura découvert dans la dialectique même du refoulement : “Nous ne manquons jamais de découvrir qu'un souvenir refoulé ne s'est transformé qu'après coup [nachträglich] en traumatisme". Cette simple découverte emporte tout avec elle. Désormais, l'origine ne pourra plus se réduire à une source factuelle, quelle que soit son "antiquité" chronologique (puisque c'est une image de mémoire qui, après coup, prend valeur de traumatisme) $»^{44}$.

31 À proprement parler, ce n'est donc pas l'événement originaire (entendu comme un factum traumatique historiquement situé en un point précis du passé) qui se répète dans le symptôme : il n'est cet événement que dans l'après-coup (ex-post, pour ainsi dire), devenant à son tour symptôme qui "dérive" et, à sa manière, réitère. L'événement luimême devient ainsi, ultérieurement, l'origine dont le symptôme est compris comme le futur, un futur «causé» par tel ou tel fait passé.

\section{Mouvements rétrogrades}

Au-delà du rapprochement de la conception freudienne de la Nachträglichkeit et de l'idée de la répétition des différences élaborée par Deleuze (par le bergsonien Deleuze ${ }^{45}$ ), il ne nous semble pas incongru de souligner la proximité entre la théorie de l'origine figurale de Warburg et la doctrine de la vérité proposée par un de ses contemporains célèbres, Henri Bergson, penseur qui ne manqua d'attirer l'attention de l'historien de l'art (comme l'indiquent les notes "Bergson " du Zettelkasten, n. 51, conservé dans les archives Warburg de Londres). 

écrit en 1896 et dont le troisième chapitre est significativement intitulé " De la survivance des images ") revient, dans la première partie de La pensée et le mouvant intitulée Croissance de la vérité. Mouvement rétrograde du vrai, à des réflexions consignées quelques années auparavant, en 1913, à l'occasion de deux conférences données à la Columbia University de New York ${ }^{46}$. Tout en parcourant les thèmes fondateurs de sa propre philosophie (le rapport entre l'espace et le temps, les notions de durée et d'intuition, les catégories de possibilité et de mouvement), Bergson souligne l'existence d'une singulière «logique de rétrospection $»^{47}$ qu'il caractérise ainsi : «Les choses et les événements se produisent à des moments déterminés; le jugement qui constate l'apparition de la chose ou de l'événement ne peut venir qu'après eux ; il a donc sa date. Mais cette date s'efface aussitôt, en vertu du principe, ancré dans notre intelligence, que toute vérité est éternelle. Si le jugement est vrai à présent, il doit, nous semble-t-il, l'avoir été toujours. Il avait beau n'être pas encore formulé : il se posait lui-même en droit, avant d'être posé en fait. À toute affirmation vraie nous attribuons ainsi un effet rétroactif ; ou plutôt nous lui imprimons un mouvement rétrograde» ${ }^{48}$. possibilité la réalité (comme si idée et chose, possibilité et réalité ne se produisaient pas au même moment), implique, selon Bergson, des conséquences qu'il n'hésite pas à dire «innombrables». Nous envisagerons ici seulement celle qui paraît directement pertinente au regard de la perspective «archéologique» de la théorie warburgienne de l'origine des images. Voyons l'«exemple simple» proposé par Bergson d'un mouvement rétrograde observable dans l'histoire de la culture : «Rien ne nous empêche aujourd'hui de rattacher le romantisme du dix-neuvième siècle à ce qu'il y avait déjà de romantique chez les classiques. Mais l'aspect romantique du classicisme ne s'est dégagé que par l'effet rétroactif du romantisme une fois apparu. S'il n'y avait pas eu un Rousseau, un Chateaubriand, un Vigny, un Victor Hugo, non seulement on n'aurait jamais aperçu, mais encore il n'y aurait réellement pas eu de romantisme chez les classiques d'autrefois, car ce romantisme des classiques ne se réalise que par le découpage, dans leur œuvre, d'un certain aspect, et la découpure, avec sa forme particulière, n'existait pas plus dans la littérature classique avant l'apparition du romantisme». De cette manière, dit Bergson, le romantisme a créé rétroactivement «sa propre préfiguration dans le passé, et une explication de lui-même par ses antécédents» ${ }^{49}$.

Préfiguration : rappelons-nous que dans son essai sur Manet, Warburg parlait de «fonction pré-figurante» (vorprägende Funktion) des divinités mythologiques pour le tableau du XIXe siècle. Une telle préfiguration est, selon Bergson, une «illusion»" ${ }^{50}$, mais le seul fait qu'il en admette d' «innombrables» conséquences nous suggère qu'il faut tenir compte de son efficacité.

Un auditeur des leçons bergsoniennes à la Sorbonne destiné à un grand avenir littéraire, Thomas Stearns Eliot ${ }^{51}$, a développé une doctrine de la poésie qui comprend le rapport avec le passé comme un rapport actif dans la mesure où le passé lui-même est actuel. Son célèbre essai de 1919, Tradition et talent individuel, livre une conception de l'histoire de la littérature entendue comme un champ formant système et pensée à la lumière du rapport entre présent et passé : «Les monuments existants forment entre eux un ordre idéal que modifie l'introduction de la nouvelle (vraiment "nouvelle") œuvre d'art. L'ordre existant est complet avant que n'arrive l'œuvre nouvelle ; pour que l'ordre subsiste après l'addition de l'élément nouveau, il faut que l'ordre existant 
tout entier soit changé, si peu que ce soit ; et les rapports, les proportions, les valeurs de chaque œuvre d'art par rapport à l'ensemble sont ainsi rajustés ; et c'est en ceci que l'ancien et le nouveau se conforment l'un à l'autre. Quiconque a admis cette idée de l'ordre, de la forme de la littérature européenne, de la littérature anglaise, ne trouvera pas absurde que le passé soit modifié par le présent, tout autant que le présent est dirigé par le passé» ${ }^{52}$.

Le passé, donc, n'est pas une dimension temporelle repliée sur elle-même, il n'est pas constitué d'un ensemble de faits qui auraient eu lieu une fois pour toutes et qui détermineraient mécaniquement, le long d'une rigoureuse chaîne, une série d'effets successifs inévitables, procédant de causes antécédentes. Le passé, bien au contraire, est ouvert au présent, il détermine le présent autant qu'il est déterminé par lui, dans un rapport de corrélation et de réciprocité.

Lecteur sagace d'Eliot et de Bergson, Jorge Luis Borges a tiré la conséquence la plus rigoureuse de ces prémisses : dans l'essai de 1951 dédié par l'écrivain argentin à Kafka et ses précurseurs, on apprend que ceux qui préfigurent Kafka, comme le philosophe Zénon, le prosateur chinois du $9^{\text {ème }}$ siècle Han Yu, Kierkegaard, Robert Browning, Léon Bloy et Lord Dunsany, sont tout à fait irréductibles les uns aux autres. «Si je ne me trompe pas - remarque Borges -, les textes disparates que je viens d'énumérer ressemblent à Kafka, mais ils ne se ressemblent pas tous entre eux. Ce dernier fait est le plus significatif. Dans chacun de ces morceaux se trouve, à quelque degré, la singularité de Kafka, mais si Kafka n'avait pas écrit, personne ne pourrait s'en apercevoir. À vrai dire, elle n'existerait pas $»^{53}$. Ces auteurs, si différents l'un de l'autre, l'œuvre kafkaïenne ne se limite pas à les évoquer, à les citer, à y faire allusion ; son œuvre les institue littéralement ex-post, après coup, comme ressemblants, en fait les éléments d'un système, les membres d'une classe. L'écriture de Kafka, non seulement crée la tradition qui la suivra (mouvement antérograde), conformément à notre habituelle perspective historiciste, mais encore - et peut-être surtout - la tradition qui la précède (mouvement rétrograde). On pourrait, à ce propos, parler d'un plagiat " a priori " ou " par anticipation $»^{54}$.

Si elle veut parvenir à saisir la temporalité complexe qui gouverne ces phénomènes, l'histoire de la culture (et l'histoire des images qui en constitue un chapitre fondamental, ainsi que Warburg l'a posé), ne peut se permettre de considérer ce double mouvement comme un simple paradoxe, ni le traiter comme une pure illusion. Elle doit, au contraire, prendre pleinement en charge l'incessante dialectique entre pré- et post-formation, en se débarrassant de tout historicisme naïf et de toute croyance ingénue en la possibilité d'une absolue nouveauté.

\section{NOTES}

1. Je tiens à remercier Sabine Forero Mendoza dont les remarques bienveillantes et précieuses furent décisives pour la mise en forme finale de ce texte. 
2. Voir les essais recueillis dans Il Cézanne degli scrittori, dei poeti e dei filosofi, éd. par G. Cianci, E. Franzini et A. Negri, Milano, Edizioni Bocca, 2001.

3. P. Valéry, Triomphe de Manet (1932), dans Pièces sur l'art, Paris, Gallimard, 1946, pp. 161-175, ici p. 166.

4. Ch. Baudelaire, Lettre à Manet du 11 mai 1865, dans Correspondance, éd. par C. Pichois, 2 vols., Paris, Gallimard, 1973, vol. II, p. 497. Sur le rapport entre les deux, voir M. Fried, Painting Memories: On the Containment of the past in Baudelaire and Manet, "Critical Inquiry », 10/3, 1984, pp. 510-542 ; J. A. Hiddleston, Baudelaire, Manet, and Modernity, " The Modern Language Review ", 87/3, 1992, pp. 567-575.

5. E. Zola, Edouard Manet (1867), dans Mes Haines. Causeries littéraires et artistiques, Paris, Charpentier, 1879, p. 352.

6. Ibid., pp. 355-356.

7. Ibid., p. 346.

8. Ibid.

9. S. Mallarmé, The Impressionists and Edouard Manet, « The Art Monthly Review and Photographic Portfolio ", 9, 1876, pp. 117-122. Voir la traduction française par P. Verdier, Les impressionnistes et Édouard Manet, dans Les Écrivains devant l'impressionnisme, éd. par D. Riout, Paris, Macula, 1989, pp. 93-104.

10. M. Foucault, La peinture de Manet, conférence donnée à Tunis, le 20 Mai 1971 ; résumé avec illustrations : http://foucault.info/documents/manet/.

11. G. Bataille, Manet (1955), dans Euvres complètes, vol. 9, Paris, Gallimard, 1979, pp. 103-167, ici p. 115.

12. A. Malraux, Les voix du silence (1951), dans Ecrits sur l'art, 2 vols., Paris, Gallimard, 2004, vol. I, pp. 301-302.

13. Ibid., pp. 300 et 298 .

14. C. Greenberg, Modernist Painting (1960), ( http://www.sharecom.ca/greenberg/ modernism.html), p. 2.

15. M. Fried, Manet's Sources, "Artforum », 7, 1969, pp. 28-82 ; repr. in Manet's Modernism, or, the Face of Painting in the 1860s, Chicago, The University of Chicago Press, 1996, p. 505, note 224.

16. R. Krauss, Antivision, « October », 36, 1986, pp. 147-154, ici p. 147.

17. Ibid., p. 151.

18. Ibid., p. 147.

19. Ibid., p. 152.

20. Y.-A. Bois, The Use Value of « Formless », dans Y.-A. Bois-R. Krauss, Formless. A User's Guide, New York, Zone Books, 1997, p. 21.

21. G. Bataille, Manet, op. cit, p. 143.

22. Ibid.

23. Ibid., p. 145.

24. E. Chesneau, L'Art et les artistes modernes en France et en Angleterre, Paris, Didier, 1864, p. 190, note 1.

25. G. Pauli, Raffael und Manet, « Monatshefte für Kunstwissenschaft », 1, 1908, pp. 53-55. L'article de Pauli fut immédiatement présenté aux lecteurs français par Jean Meryem, qui en donna une synthèse dans sa note Raphäel et Manet, «L'Art et les Artistes », 3, 1908, pp. 24-25. Sur le rapport entre Manet et Raimondi voir B. Farwell, Manet's "Espada" and Marcantonio, "Metropolitan Museum Journal ", 2, 1969, pp. 197-207; M. Centanni, Ninfa impertinente: Victorine e la Patera di Parabiago (a proposito dei modelli del "Déjeuner sur l'herbe" di Manet e, prima, di Raffaello), « La Rivista di Engramma ", 36, 2004 (http://www.engramma.it/engramma_v4/rivista/saggio/ 36/036_manet_saggio.html).

26. A. Warburg, Le " Déjeuner sur l'herbe » de Manet. La fonction préfiguratrice des divinités élémentaires païennes pour l'évolution du sentiment moderne de la nature (1929), trad. française par S. Zilberfarb 
dans Miroirs de faille. À Rome avec Giordano Bruno et Edouard Manet, 1928-29, éd. par M. Ghelardi, Paris, Les presses du réel, 2011, p. 126.

27. Voir É. Moreau-Nélaton, Manet raconté par lui-même, 2 vols., Paris, Laurens, vol. I, pp. 48-49; A. Proust, Edouard Manet. Souvenirs, Paris, Laurens, 1913, p. 43.

28. A. Warburg, Le « Déjeuner sur l'herbe » de Manet, op. cit., p. 126.

29. On peut retrouver cette constellation iconographique sur la planche 55 [Fig.5] de l'Atlas (voir A. Warburg, Der Bilderatlas Mnemosyne, éd. par M. Warnke, Berlin, Akademie Verlag, 2000).

30. A. Warburg, Le « Déjeuner sur l'herbe » de Manet, op. cit., p. 126.

31. A ce propos, voir H. Damisch, Le jugement de Paris : iconologie analytique 1 , éd. revue et augmentée, Paris, Flammarion, 1997 (avec référence à Warburg interprète de Manet, pp. 199-211).

32. Warburg renvoie au catalogue réalisé par C. Robert, Die antiken Sarkophagreliefs, Berlin, Grote Gebr. Mann, 1890, vol. II, pp. 13-20, ill. IV-V. On peut retrouver les deux sarcophages sur la planche 4 [Fig. 8] de l'Atlas (op. cit.)

33. O. Jahn, Über einige Darstellungen des Parisurtheils, «Berichte über die Verhandlungen der Königlich Sächsischen Gesellschaft der Wissenschaften zu Leipzig. Philologisch-Historische Klasse », 1849, vol. I, pp. 55-69.

34. A. Springer, Raffael und Michelangelo, Leipzig, E.A. Seemann, 1878, pp. 310-311.

35. H. Thode, Die Antiken in den Stichen Marcanton's, Agostino Veneziano's und Marco Dente's, Leipzig, E.A. Seemann, 1881, p. 25.

36. J. von Sandrart, Teutsche Akademie der edlen Bau-, Bild- und Mahlerey-Künste, Nürnberg 1675, II, Buch II ("Italienische Künstler"), p. 205 (http://ta.sandrart.net/edition/text/view/420? query=paris\&truncation=\#querystring1).

37. A. Warburg, Le « Déjeuner sur l'herbe » de Manet, op. cit., p. 126.

38. Ibid., p. 128.

39. Ibid., p. 130.

40. Ibid., p. 133.

41. Ibid.

42. G. Deleuze, Différence et répétition (1968), 7 $7^{\mathrm{ème}}$ éd., Paris, PUF, 1993, p. 145.

43. G. Didi-Huberman, L'image survivante. Histoire de l'art et temps de fantômes selon Aby Warburg, Paris, Minuit, 2002, p. 332.

44. Ibid., p. 331. La citation de Freud est tirée de l'Esquisse d'une psychologie scientifique (1895), dans La Naissance de la psychanalyse, Paris, PUF, 1973, p. 366.

45. Voir, G. Deleuze, Le bergsonisme, Paris, PUF, 1966.

46. $\mathrm{H}$. Bergson, Croissance de la vérité. Mouvement rétrograde du vrai, dans La pensée et le mouvant (1934), Paris, PUF, 1969, pp. 6-17. Dans la note 1, p. 13 de cette édition, Bergson renvoie aux conférences données en 1913 à la Columbia University sous le titre Spiritualité et liberté et The Method of Philosophy, an outline of a Theory of Knowledge, conférences dont quelques extraits sont publiés dans Mélanges, Paris, PUF, 1972, pp. 975-989.

47. H. Bergson, Croissance de la vérité. Mouvement rétrograde du vrai, op. cit., p. 15.

48. Ibid., p. 13.

49. Ibid., p. 14.

50. Ibid., p. 13.

51. Dans les archives T.S. Eliot conservées à la Harvard University, sous le numéro d'inventaire « Series: IV. Notes, odd letters, trivia, etc. ", sont recueillies, au numéro 130, des annotations relatives aux conférences de Bergson données à Paris en 1910-1911 et au numéro 132, le manuscrit d'un texte sur Bergson, contemporain aux annotations. À propos de l'influence exercée par Bergson sur Eliot, voir Ph. Le Brun, T. S. Eliot and Henri Bergson, "The Review of English Studies », 18, 70-71, 1967, pp. 149-161 et pp. 274-286 ; P. Douglass, Bergson, Eliot, and American Literature, Lexington-KY, University Press of Kentucky, 1986. 
52. T.S. Eliot, La Tradition et le talent individuel (1919), dans Essais choisis, trad. française par H. Fluchère, Paris, Seuil, 1950 ; rééd. 1999, p. 29.

53. J. L. Borges, Kafka et ses précurseurs (1951), dans Autres Inquisitions, Euvres complètes, Paris, Gallimard, 1993, vol. I, p. 751.

54. A. Pinotti, Citazioni a posteriori e plagi a priori. Borges, Eliot, Warburg, Goethe, «Leitmotiv ", 2, 2002, pp. 37-43 (http://www.ledonline.it/leitmotiv/allegati/leitmotiv020204.pdf); P. Bayard, Le plagiat par anticipation, Paris, Minuit, 2009.

\section{INDEX}

Mots-clés : Manet, césure, peinture, XIX siècle, théorie, interprétation, Déjeuner sur l'herbe

\section{AUTEUR}

\section{ANDREA PINOTTI}

Professeur associé d'Esthétique à l'Université de Milan, il s'interesse en particulier à la relation entre esthétique, philosophie de l'art et histoire de l'art dans la culture de langue allemande du XIX et XX siècle. Fellow d'institutions comme l'Italian Academy for Advanced Studies at Columbia University, le Warburg Institute à Londres, le ZfL à Berlin, il est Directeur de Programme au Collège International de Philosophie à Paris (2011-2016). Parmi ses publications: Le corps du style. Histoire de l'art comme histoire de l'esthétique à partir de Semper, Riegl, Wölfflin (Il corpo dello stile. Storia dell'arte come storia dell'estetica a partire da Semper, Riegl, Wölfflin, Supplementa Preprint, Aesthetica ed., Palermo $1998 ; 2^{\text {ème }}$ éd. Mimesis, Milan 2001); Petite histoire de la distance. Walter Benjamin historien de la perception (Piccola storia della lontananza. Walter Benjamin storico della percezione, Libreria Raffaello Cortina, Milan 1999); Mémoires du neutre. Morphologie de l'image chez Aby Warburg (Memorie del neutro. Morfologia dell'immagine in Aby Warburg, Mimesis, Milan 2001); Tableau et type. L'esthétique chez Burckhardt (Quadro e tipo. L'estetico in Burckhardt, Il Castoro, Milan 2004); Esthétique de la peinture (Estetica della pittura, il Mulino, Bologne 2007); Le revers de l'image. Droite et gauche dans l'art (Il rovescio dell'immagine. Destra e sinistra nell'arte, Tre Lune, Mantoue 2010); Empathie. Histoire d'une idée de Platon au post-humain (Empatia. Storia di un'idea da Platone al postumano, Laterza, Rome-Bari 2011). 